\title{
A simple high-voltage high current spark gap with subnanosecond jitter triggered by femtosecond laser filamentation
}

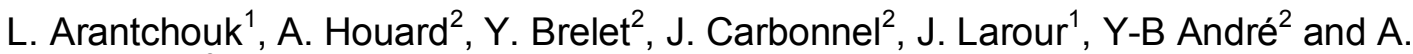 \\ Mysyrowicz ${ }^{2}$ \\ ${ }^{I}$ Laboratoire de Physique des Plasmas, Ecole Polytechnique, CNRS, 91128 Palaiseau, France \\ ${ }^{2}$ Laboratoire d'Optique Appliquée, ENSTA ParisTech, Ecole Polytechnique, CNRS, 91762 Palaiseau, \\ France
}

\begin{abstract}
We describe a simple, sturdy and reliable spark gap operating with air at atmospheric pressure and able to switch currents in excess of $10 \mathrm{kA}$ with sub-nanosecond jitter. The spark gap is remotely triggered by a femtosecond laser filament.
\end{abstract}

Many applications rely on the initiation of a well controlled discharge between two charged electrodes. For high current applications, a gas-filled trigger is necessary. The breakdown of the gas filling the inter-electrode gap D is obtained by the introduction of an important electric field perturbation between the electrodes. This perturbation is achieved either by a high voltage (HV) trigger pulse through a third electrode, or by illumination of the inter-electrode region with a laser pulse. An outstanding feature of laser triggering is the absence of electrical coupling between the discharge electrodes and the triggering circuit, leading to a safe mode of operation.

Since the first laser-triggered switching experiments in the early 1960s, a wide variety of spark gap geometries and laser types have been investigated ${ }^{1}$. It turned out that focusing a laser axially rather than transversally inside the gap along the electrodes gave a better temporal stability of the switched pulse. Using nanosecond laser pulses, several authors have reported the switching of hundreds of $\mathrm{kV}$ across an air filled gap of $\mathrm{cm}$ length with a nanosecond jitter time $\mathrm{e}^{2-7}$. The jitter could be reduced to hundred of picoseconds with a $\mathrm{SF}_{6}$ filled cell ${ }^{8}$. Using femtosecond laser pulses, other authors even achieved a jitter in the range of tens of picoseconds in air at atmospheric pressure but with significantly lower operating voltage $(4.5 \mathrm{kV})$ in a mm wide gap ${ }^{9}$.

In this paper we present results on atmospheric air spark gap triggering with help of femtosecond laser filamentation. Triggering via filamentation has several advantages. Since filament generated plasma column can be created at a distance of several hundreds of meters from the laser, remote triggering can be achieved. Because filaments have a near constant longitudinal electron density over several meters, they connect the two electrodes quasi-instantaneously, circumventing the complex process required in the case of an initial localized plasma, and reducing thereby the jitter. Thus, this spark gap combines the advantages of large inter-electrode air gaps capable of switching high voltages and the low jitter of narrow spark gaps or $\mathrm{SF}_{6}$ filled cells. Main disadvantage is the requirement of a high power femtosecond laser, a commercially available but at the moment still rather expensive piece of equipment.

Filamentation is the result of a dynamical competition between two main effects. The Kerr effect produces laser beam self-focusing while multi-photon ionization creates a plasma, which tends to defocus the laser beam ${ }^{10}$. Due to the dynamic competition between these two effects, the laser beam enters a dynamic self-guiding regime provided the initial laser peak power slightly exceeds a critical threshold $\mathrm{P}_{\mathrm{cr}}$. $\left(\mathrm{P}_{\mathrm{cr}} \approx 5 \mathrm{GW}\right.$ in atmospheric air for a 50 fs laser pulse at $\left.\lambda_{0}=800 \mathrm{~nm}\right)$. Dynamic selfguiding gives rise to a plasma column of diameter $\sim 100 \mu \mathrm{m}$ stretching over several meters with an electron density of $\sim 10^{16} \mathrm{~cm}^{-3}$. For $\mathrm{P}>>\mathrm{P}_{\text {cr }}$, a large number $\mathrm{N} \sim \mathrm{P} / \mathrm{P}_{\text {cr }}$ of parallel filaments are produced. A mobile Ti:Sapphire laser chain called ENSTAmobile was used in our experiments. This laser delivers pulses at a central wavelength $\lambda_{0}=800 \mathrm{~nm}$ with a minimum duration of $50 \mathrm{fs}$, an energy up to $300 \mathrm{~mJ}$ at a repetition rate of $10 \mathrm{~Hz}$. In our experiments, the laser pulse of energy $\mathrm{E}=200-250$ $\mathrm{mJ}$ was linearly chirped to a duration of $\tau=700$ fs corresponding to an input peak power of $360 \mathrm{GW}$, far above the critical power. In this regime the laser beam breaks up into about 50 parallel filaments. The beam was focused in air with a $5 \mathrm{~m}$ focal length lens leading to a merging of these filaments into a quasi-homogeneous plasma column with an effective length of $2 \mathrm{~m}$ and a diameter of $\sim 3 \mathrm{~mm}$, 
extending on both sides of the geometrical focus. The experimental spark gap was installed in the middle of the plasma column, close to the geometrical focus of the laser beam.

Figure 1 gives a schematic overview of the experimental setup. The gap switch consisted of two cylindrical stainless electrodes of face diameter $36 \mathrm{~mm}$ with holes drilled in their center.We have operated the spark gap for more than 5000 laser shots, without any obvious deterioration of its characteristics. . Results presented here were obtained with holes of $3 \mathrm{~mm}$ diameter, allowing $90 \%$ of laser energy to pass through. The chosen hole diameter optimized the contact between the laser created plasma column and the electrodes and therefore the performances of the spark gap. A storage capacitor of nominal value $\mathrm{C}=360 \mathrm{nF}$ was first charged up to a voltage $\mathrm{U}_{0} \leq 30 \mathrm{kV}$. The high voltage was applied to the spark gap set in parallel with a $50 \Omega$ resistance by activating a conventional switch T. It created on the spark gap electrodes a voltage pulse with rise time $(10 \%-90 \%) \sim 50 \mathrm{~ns}$ and exponential decay $\tau=\mathrm{RC} \approx 14 \mu \mathrm{s}$. After the closing of the spark gap the LC circuit produced exponentially damped current oscillations with a half period $\mathrm{T} / 2 \approx 2 \mu \mathrm{s}$. The current maximal amplitude reached $\sim 12.5 \mathrm{kA}$ with a $30 \mathrm{kV}$ charge.

The self-breakdown threshold in static regime was measured to be close to the Paschen Law for atmospheric air $U_{c r}=2.4 D+6.7 \sqrt{0.1 D}$, where $U_{c r}$ is in $\mathrm{kV}$ and the gap $D$ is in millimeters. The pulsed operation raises the DC breakdown limit by $20-28 \%$, allowing us to study the laser triggering in a larger voltage region around the point of the statistical self-breakdown. The high voltage signal on the switch gap electrodes was measured by a resistive divider (see Fig. 1) and the current by a Pulse Current Transformer (Model Stangenes 0.5-0.01). The onset of discharge was defined as the time for which the voltage reached $60 \%$ of the voltage maximum.. The laser arrival time was detected by a fast photodiode together with a 1-GHz bandwidth oscilloscope, (LeCroy Wavepro). Each measurement point was averaged over about 10 shots. Typical signals of the laser pulse and the gap switch voltage are presented in Fig. 2.The triggered laser beam was typically sent 400-500 ns after the onset of voltage on the switch $(\mathrm{T})(\mathrm{t}=0 \mathrm{~s}$ in Fig. 2). We checked that the spark gap switching characteristics did not depend on this delay over the range 250-1200 ns. The evolution of the delay between the triggered laser and the start of the discharge is presented in Fig. 3 as a function of the normalized breakdown voltage $\mathrm{U}_{\text {norm }}=\mathrm{U} / \mathrm{U}_{\mathrm{cr}}$ for a set of inter-electrode gaps comprised between 7 and $10 \mathrm{~mm}$ (D7-D10). The experimental points lie around the curve Fit $(n s)=17 \times\left(U / U_{c r}\right)^{-1.55}$.

An important parameter of the spark gap is the jitter, which is usually defined as the standard deviation of the switching time delay (Fig. 4). One can see that it is possible to have a jitter of 0.2-0.4 ns in the region $0.7<\mathrm{U}_{\text {norm }}<1.2$ with a $10 \mathrm{~mm}$ gap. The measured jitter is close to the time resolution of the oscilloscope ( $1 \mathrm{GHz}$ and $10 \mathrm{GSa}$ ).

We also studied a situation where the laser beam arrives into the inter-electrode gap before applying the voltage ${ }^{11}$. It allows producing a high voltage pulse of controlled duration. The voltage pulse beginning is defined at $20 \%$ of the voltage maximum and the breakdown beginning at $75 \%$ level. Figure 5 shows typical signals obtained with the $8-\mathrm{mm}$ gap charged up to $29 \mathrm{kV}$ and with the delay between the laser and the voltage application of about $700 \mathrm{~ns}$. With the $8 \mathrm{~mm}$ gap the pulse duration changed in the range 30-50 ns when the laser delay varied between 0.7-2 $\mu$ s under the gap voltage $29 \mathrm{kV}$. With the $10 \mathrm{~mm}$ gap the pulse duration changed in the range 75-85 ns when the laser delay varied between 3.5 - $4.7 \mu$ s under the same gap voltage $29 \mathrm{kV}$.

Operation with $15-\mathrm{mm}$ gap and maximum applied voltage $30 \mathrm{kV}$ allowed breakdown with $100 \%$ probability but the pulse width varied strongly from shot to shot. It ranged from 300 to $600 \mathrm{~ns}$ with the laser delay 200-300 ns and from 700 to $3500 \mathrm{~ns}$ with the laser delay about $3 \mu \mathrm{s}$.

In summary, we have demonstrated the operation of a simple, easily reconfigurable spark gap operating with ambient air. When remotely triggered by the plasma from filamentation of femtosecond laser pulses, it is capable to commute reliably high voltage in the range of several tens of $\mathrm{kV}$ and peak currents in the range of kA. With optimized electrodes, the jitter is $0.2-0.4 \mathrm{~ns}$, close to the time resolution of our detection. The spark gap can also operate as a high voltage pulse generator of tunable duration. 


\section{ACKNOWLEDGMENTS}

This work was supported by the French Direction Générale de 1'Armement (grant EPAT n²007 95091). The authors would like to thank Dr. B. Forestier, A. Dos Santos and J.-L. Charles for their technical help.

\section{REFERENCES}

${ }^{1}$ A. H Guenther and J. R. Bettis, J. Phys. D: Appl. Phys. 11, 1577 (1978); $5^{\text {th }}$ Int. Pulsed Power Conf. 47 (1985).

${ }^{2}$ J. R. Woodworth, R. G. Adams, and C. A. Frost, IEEE Trans. Plasma Sci. PS-10, 257 (1982).

${ }^{3}$ B. T. Hutsell, S. D. Kovaleski, D. L. Sullivan and J. M. Gahl, IEEE Trans. Dielectrics Electrical Insulation 16, 999 (2009).

${ }^{4}$ K. R. LeChien, M. E. Savage, V. Anaya, D. E. Bliss, W. T. Clark, J. P. Corley, G. Felts, J. E. Garrity, D. W. Guthrie, K. C. Hodge, et al., Phys. Rev. Special Topics-Accelerators and Beams 11, $060402(2008)$.

${ }^{5}$ W. T. Clark, M. E. Savage, D. E. Bliss, B. S. Stoltzfus, and J. R. Woodworth, IEEE 34th International Conference on Plasma Science 195 (2007).

${ }^{6}$ J. A. Miles, S. F. Adams, C. A DeJoseph, and A. C. Laber, Proceedings of IEEE International Power Modulators and High Voltage Conference, 299 (2008).

${ }^{7}$ N. L. Aleksandrov and E. M. Bazelyan, Plasma Physics Reports 27, 1057 (2001).

${ }^{8}$ J. R. Woodworth, C. A. Frost and T. A. Green, J. Appl. Phys. 53, 4734 (1982).

${ }^{9}$ J. Hendriks, B. H. P. Broks, J. J. A. M. Van der Mullen, and G. J. H. Brussaard, J. Appl. Phys. 98, 043309 (2005).

${ }^{10}$ A. Couairon and A. Mysyrowicz, Phys. Rep. 441, 47 (2007).

${ }^{11}$ B. La Fontaine, F. Vidal, D. Comtois, C.-Y. Chien, A. Desparois, T. W. Johnston, J. C. Kieffer, H.

P. Mercure, H. Pepin, and F. A. M. Rizk, IEEE Trans. Plasma Sci. 27, 688 (1999). 


\section{Figures}

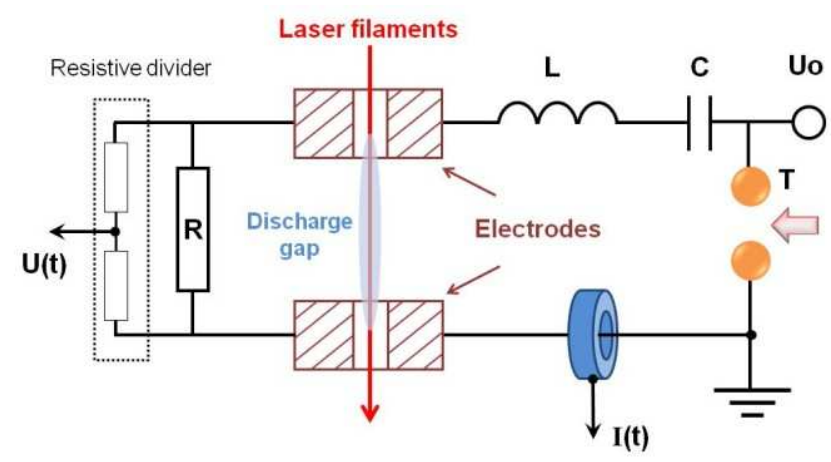

FIG. 1. (Color online) Schematic view of the experimental set-up with a pulsed voltage applied to the axial gap switch. The laser beam passes through the axial holes in the electrodes.

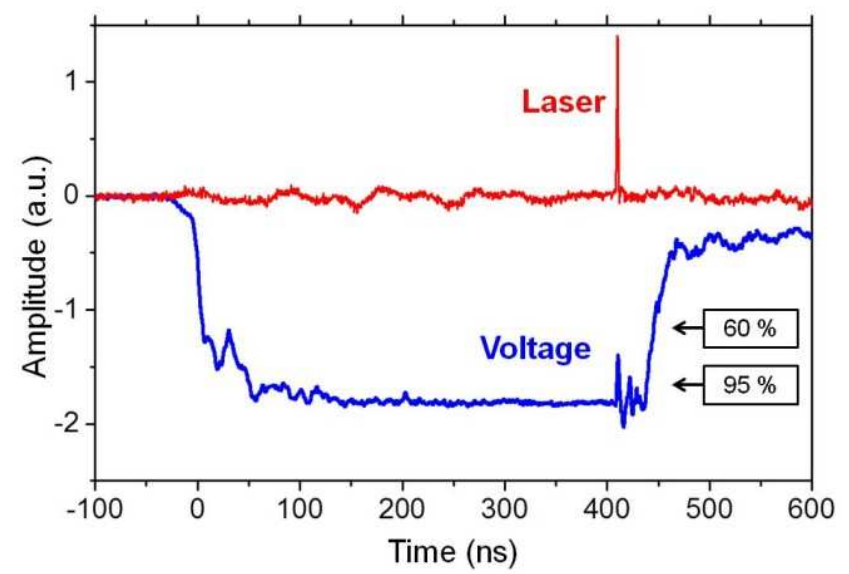

FIG. 2. (Color online) Typical signals of the laser beam and the gap switch voltage.

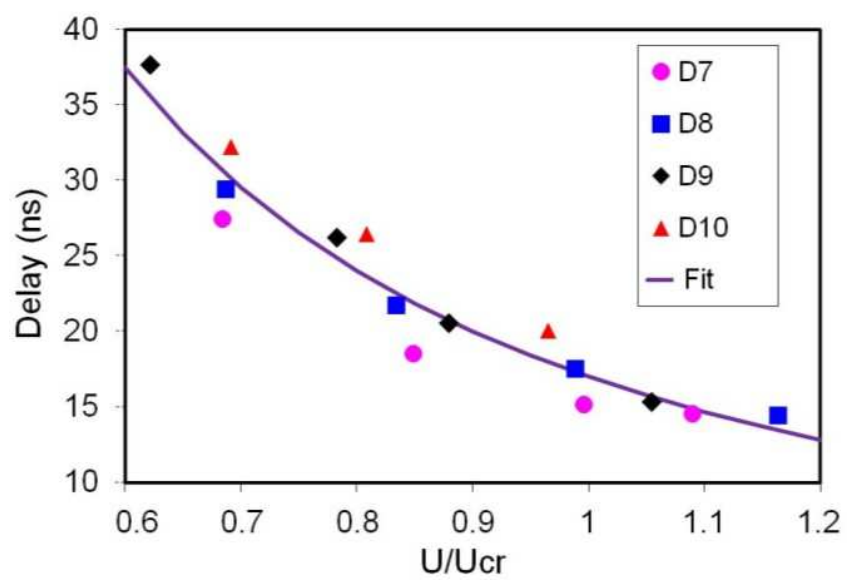

FIG. 3. (Color online) Delay between the triggered laser and the discharge beginning for interelectrode gaps 7 (D7) to $10 \mathrm{~mm}$ (D10) versus the voltage on the switch T with the hole $\varnothing 3 \mathrm{~mm}$ and fitting curve. 


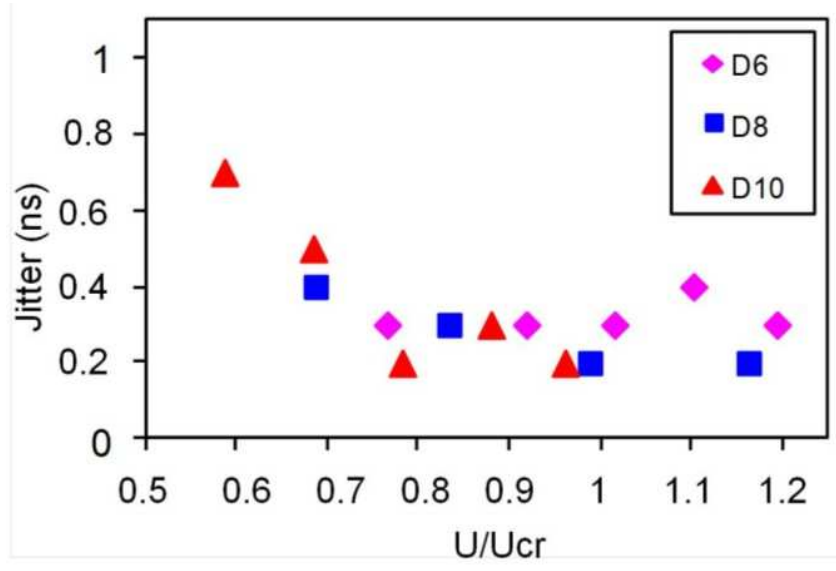

FIG. 4. (Color online) Jitter for the gap switch with the hole $Ø 3 \mathrm{~mm}$ and gaps 6 (D6) to 10 (D10) $\mathrm{mm}$.

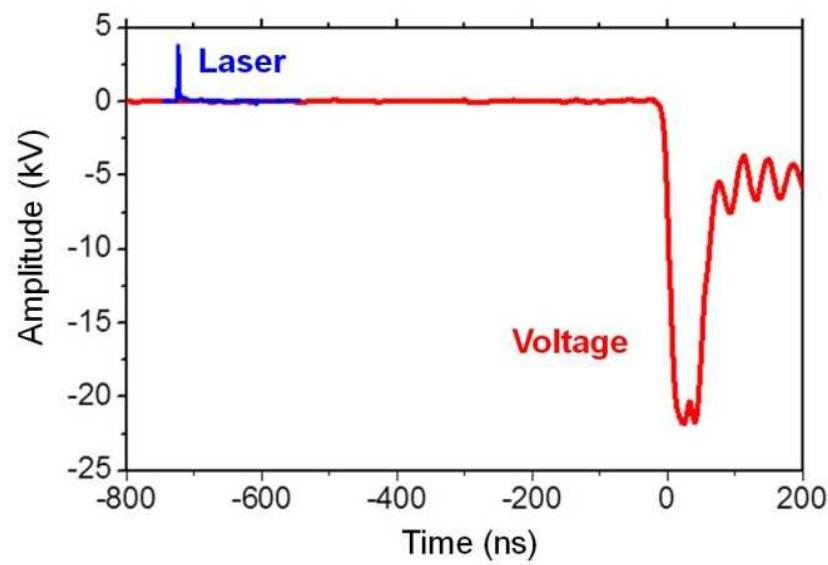

FIG. 5. (Color online) Triggering with the laser arrival before the application of the voltage pulse. 